\title{
Antiferromagnetic ordering of energy levels for spin ladder with four-spin cyclic exchange: Generalization of the Lieb-Mattis theorem
}

\author{
Tigran Hakobyan* \\ Yerevan State University, 1 Alex Manoogian, 375025 Yerevan, Armenia, \\ Yerevan Physics Institute, 2 Alikhanyan Br., 375036 Yerevan, Armenia
}

(Dated: February 17, 2008)

\begin{abstract}
The Lieb-Mattis theorem is generalized to an antiferromagnetic spin-ladder model with four-spin cyclic exchange interaction. We prove that for $J>2 K$, the antiferromagnetic ordering of energy levels takes place separately in two sectors, which remain symmetric and antisymmetric under the reflection with respect to the longitudinal axis of the ladder. We prove also that at the self-dual point $J=2 K$, the Lieb-Mattis rule holds in the sectors with fixed number of rung singlets. In both cases, it agrees with the similar rule for Haldane chain with appropriate spin number.
\end{abstract}

The systems with multi-spin exchanges have gained a lot of interest for a long time (for a recent review, see Refs. 1|2). These interactions arise at higher orders of a strong coupling expansion of a half-filled Hubbard model and provide perturbative corrections to the Heisenberg model. $\underline{3}$ The multi-spin cyclic permutations first were suggested to be important in two-dimensional (2D) quantum solids like ${ }^{3} \mathrm{He} . \underline{\underline{4}}$ The relevance of the four-spin cyclic interaction around square plaquettes of $\mathrm{CuO}_{2}$ planes in high-temperature superconductors was suggested in Ref. 5, and then it was proven experimentally $\frac{1}{\underline{1}}$ Recently, their quasi-1D counterparts with similar structure and properties have been studied intensively (see Ref. 6 for a review). Note that the spin-ladder model is the simplest system, where the four-spin cyclic exchange appears from the electron interaction. In fact, the investigations of some copper-based spin-ladder materials have revealed the relevance of four-spin exchange term 7 (see Ref. 8 for a review). The ground-state phase diagrams of frustrated

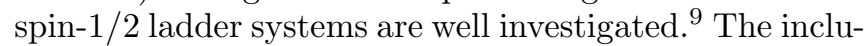
sion of four-spin interactions may result in new unconventional phases $10,11,12,13$ The different phases are related by a duality transformation $\underline{12}$

In this paper, we will generalize the well-known LiebMattis theorem on ordering of energy levels to the ladder model with four-spin cyclic interaction. For finite-size Heisenberg models on bipartite lattices, Lieb and Mattis proved that the lowest energy $E(S)$ in the sector, where the total spin is equal to $S$, is a monotone increasing function of the spin for any $S \geq S_{\mathrm{gs}} \stackrel{14}{\underline{w}}$ where $S_{\mathrm{gs}}$ is the spin of the ground state. This property is known as LiebMattis theorem about antiferromagnetic ordering of energy levels. The bipartiteness means that the lattice can be divided into two sublattices $A$ and $B$, so that all interactions within the same sublattice are ferromagnetic while the interactions between different sublattices are antiferromagnetic. Moreover, the quantum ground state of finite-size system is a unique multiplet with total spin $S_{\mathrm{gs}}=\left|S_{A}-S_{B}\right|$, which coincides with the spin of the classical ground state, namely, the Néel state. Here, $S_{A}$ and $S_{B}$ are the highest possible spins on corresponding sublattices $\stackrel{14}{\underline{14}}$ In one dimension, the Lieb-Mattis theorem is valid for a more general class of quantum systems. In particular, it is true for the Hubbard chain. $\stackrel{15}{*}$ Recently, it has been generalized to $\mathrm{SU}(n)$ symmetric chain. $\frac{16}{} \mathrm{~A}$ ferromagnetic ordering of energy levels has also been formulated and proven for the Heisenberg chain $\underline{\underline{17}}$

According to numerical simulations, a weak frustration may preserve the antiferromagnetic ordering of energy levels $\frac{18}{18}$ and the ground-state spin value, $\frac{19}{\underline{t}}$ whereas a stronger frustration can violate the Lieb-Mattis property. For many frustrated systems, the lowest levels $E(S)$ show approximately parabolic or linear growth 20 So, although the Lieb-Mattis theorem is not valid for frustrated spin systems in general, it (or its proper extensions) may be valid for certain systems. In particular, its generalization to reflection-symmetric frustrated spin- $1 / 2$ ladder model has been formulated and proven recently ${ }_{21}$ Here, we obtain similar results for the frustration caused by four-spin ring interaction.

The Hamiltonian of the system reads:

$$
\begin{aligned}
H & =\sum_{l=1}^{N-1} J_{l}^{\|}\left(\mathbf{S}_{1, l} \cdot \mathbf{S}_{1, l+1}+\mathbf{S}_{2, l} \cdot \mathbf{S}_{2, l+1}\right) \\
& +\sum_{l=1}^{N} J_{l}^{\perp} \mathbf{S}_{1, l} \cdot \mathbf{S}_{2, l}+\sum_{l=1}^{N-1} K_{l}\left(P_{l, l+1}^{\square}+P_{l, l+1}^{\square-1}\right),
\end{aligned}
$$

where $\mathbf{S}_{1, l}$ and $\mathbf{S}_{2, l}$ are the spin-1/2 operators of the first and second chains respectively. The cyclic ring exchange $P^{\square}+P^{\square-1}$ is a superposition of clockwise and counter clockwise permutations of four spins around each plaquette. We consider the following range of couplings:

$$
J_{l}^{\|}>2 K_{l}>0 .
$$

The system possesses $\mathrm{SU}(2)$ spin symmetry. It has also $Z_{2}$ symmetry corresponding to the reflection with respect to the longitudinal axis. So, the Hamiltonian remains invariant on individual sectors with fixed values of spin $S$ and reflection $\sigma= \pm 1$ quantum numbers.

We will prove that for the model (11), (2), the antiferromagnetic ordering of energy levels holds independently in symmetric $(\sigma=1)$ and antisymmetric $(\sigma=-1)$ sectors and conforms to the similar rule for Haldane chain,, 22 i.e. spin-1 Heisenberg chain, with $N$ and $N-1$ spins, respectively. Namely, the lowest-energy levels $E_{\sigma}(S)$ in sectors 
with spin $S$ and parity $\sigma$ are nondegenerate and monotone increasing functions of $S$ for $S \geq S_{\mathrm{gs}}(\sigma)$. Here,

$$
S_{\mathrm{gs}}(\sigma)= \begin{cases}0, & \text { if } \sigma=(-1)^{N} \\ 1, & \text { if } \sigma=(-1)^{N-1}\end{cases}
$$

is the ground-state spin value in the sector with parity $\sigma$. The nondegeneracy means that all states on corresponding level form a unique multiplet. So, the ground state in $\sigma=(-1)^{N}$ sector is a unique singlet while in $\sigma=(-1)^{N-1}$ sector it is a unique triplet.

We will prove also that at the self-dual point $J_{l}^{\|}=$ $2 K_{l}$, the Lieb-Mattis rule holds in the sectors with fixed number of rung singlets $N_{0}$ and agrees with the similar rule for the Haldane chain with $N-N_{0}$ spins.

We begin by introducing the basis of three triplet and one singlet states for each rung:

$$
\begin{aligned}
& \left.|1\rangle=\left|\begin{array}{l}
\uparrow \\
\uparrow
\end{array}\right\rangle, \quad|\tilde{0}\rangle=\frac{1}{\sqrt{2}}\left(\begin{array}{l}
\mid \uparrow \\
\downarrow
\end{array}\right\rangle+\left|\begin{array}{l}
\downarrow \\
\uparrow
\end{array}\right\rangle\right), \quad|-1\rangle=\left|\begin{array}{l}
\downarrow \\
\downarrow
\end{array}\right\rangle, \\
& \left.|0\rangle=\frac{1}{\sqrt{2}}\left(\begin{array}{l}
\uparrow \\
\downarrow
\end{array}\right\rangle-\left|\begin{array}{l}
\downarrow \\
\uparrow
\end{array}\right\rangle\right) .
\end{aligned}
$$

Below, we will prove that all nonvanishing off-diagonal elements of the Hamiltonian (1),(2) become negative in the basis

$$
\begin{aligned}
& \left|m_{1}, \ldots, m_{N}\right\rangle \\
& \quad=(-1)^{\left[N_{0} / 2\right]+N_{0 \tilde{0}}+M_{\text {odd }}}\left|m_{1}\right\rangle \otimes \ldots \otimes\left|m_{N}\right\rangle,
\end{aligned}
$$

where $m_{l}= \pm 1, \tilde{0}, 0$ marks one of the four rung states (3). In the sing factor, $N_{0}$ is the number of singlets, and $N_{0 \tilde{0}}$ is the number of pairs $(0, \tilde{0})$ in the sequence $m_{1}, \ldots, m_{N}$, where 0 is on the left-hand side from $\tilde{0}^{21}$ $M_{\text {odd }}=\sum_{l} m_{2 l-1}$ is the total $z$-projection of odd-site spins. Note that the basic states above are eigenstates of the reflection operator with eigenvalue $\sigma=(-1)^{N_{0}}$.

First, we rewrite the Hamiltonian in a form that is more convenient for further purposes. The four-spin interaction term can be expressed via spin operators as follows (see, for instance, Ref. 23):

$$
\begin{aligned}
P_{l, l+1}^{\square} & +P_{l, l+1}^{\square-1}=\mathbf{S}_{1, l} \cdot \mathbf{S}_{1, l+1}+\mathbf{S}_{2, l} \cdot \mathbf{S}_{2, l+1}+\mathbf{S}_{1, l} \cdot \mathbf{S}_{2, l} \\
& +\mathbf{S}_{1, l+1} \cdot \mathbf{S}_{2, l+1}+\mathbf{S}_{1, l} \cdot \mathbf{S}_{2, l+1}+\mathbf{S}_{1, l+1} \cdot \mathbf{S}_{2, l} \\
& +4\left(\mathbf{S}_{1, l} \cdot \mathbf{S}_{1, l+1}\right)\left(\mathbf{S}_{2, l} \cdot \mathbf{S}_{2, l+1}\right) \\
& +4\left(\mathbf{S}_{1, l} \cdot \mathbf{S}_{2, l}\right)\left(\mathbf{S}_{1, l+1} \cdot \mathbf{S}_{2, l+1}\right) \\
& -4\left(\mathbf{S}_{1, l} \cdot \mathbf{S}_{2, l+1}\right)\left(\mathbf{S}_{2, l} \cdot \mathbf{S}_{1, l+1}\right)+1 / 4 .
\end{aligned}
$$

Using the relation $\mathbf{S}_{1} \cdot \mathbf{S}_{2}=P_{12} / 2-1 / 4$, where the operator $P_{12}$ permutes two spin states, one can present the expression above in the following form:

$$
\begin{aligned}
& P_{l, l+1}^{\square}+P_{l, l+1}^{\square-1}=2\left(\mathbf{S}_{1, l} \cdot \mathbf{S}_{2, l+1}+\mathbf{S}_{1, l+1} \cdot \mathbf{S}_{2, l}\right) \\
& +P_{l, l+1}^{\|}+P_{l, l+1}^{=}-P_{l, l+1}^{\times} .
\end{aligned}
$$

Here, $P_{l, l+1}^{\|}, P_{l, l+1}^{=}$and $P_{l, l+1}^{\times}$are, respectively, four-spin permutations along the plaquette rungs, legs and diagonals. Further, we express the two-spin interactions in terms of the symmetrized and antisymmetrized rung spin operators

$$
\mathbf{S}_{l}^{(s)}=\mathbf{S}_{1, l}+\mathbf{S}_{2, l}, \quad \mathbf{S}_{l}^{(a)}=\mathbf{S}_{1, l}-\mathbf{S}_{2, l}
$$

The operator $\mathbf{S}_{l}^{(s)}$ describes the total spin of $l$ th rung. Using (5), (6) and omitting nonessential scalar term, one can reduce the Hamiltonian (1) to the following form:

$$
\begin{aligned}
H & =\sum_{l=1}^{N-1}\left(J_{l}^{s} \mathbf{S}_{l}^{(s)} \cdot \mathbf{S}_{l+1}^{(s)}+J_{l}^{a} \mathbf{S}_{l}^{(a)} \cdot \mathbf{S}_{l+1}^{(a)}\right) \\
& +\sum_{l=1}^{N-1} K_{l}\left(P_{l, l+1}^{\|}+P_{l, l+1}^{\overline{\bar{l}}+1}-P_{l, l+1}^{\times}\right) \\
& +\sum_{l=1}^{N} \frac{J_{l}^{\perp}}{2}\left(\mathbf{S}_{l}^{(s)}\right)^{2} .
\end{aligned}
$$

In the above equation, we have introduced the symmetrized and antisymmetrized couplings

$$
J_{l}^{s}=\frac{J_{l}^{\|}}{2}+K_{l}, \quad J_{l}^{a}=\frac{J_{l}^{\|}}{2}-K_{l} .
$$

Note that a similar decomposition for the Hamiltonian without four-spin exchange was applied in Refs. 24 and 13. The permutations $P_{l, l+1}^{\|}$and $P_{l, l+1}^{\times}$have been used in Ref. 23.

The $J^{\perp}$ part of the Hamiltonian is just the sum of rung spins squares, which is diagonal in the basis (4). The local terms $P_{l, l+1}^{\|}$are also diagonal (with eigenvalues \pm 1 ), since any triplet (singlet) rung state stays symmetric (antisymmetric) under the reflection.

The $J^{s}$ terms correspond to the so-called composite spin model..$^{25}$ They conserve the spins of individual rungs because $\mathbf{S}_{l}^{(s)}$ describes the total spin of $l$ th rung. ${ }^{24}$ The singlets remain frozen at their points, and, therefore, the factor $(-1)^{\left[N_{0} / 2\right]+N_{0}}$ in (44) remains invariant. All nonvanishing off-diagonal matrix elements come from the exchanges $|\tilde{0}\rangle \otimes|\tilde{0}\rangle \leftrightarrow| \pm 1\rangle \otimes|\mp 1\rangle$ and $|\tilde{0}\rangle \otimes| \pm 1\rangle \leftrightarrow$ $| \pm 1\rangle \otimes|\tilde{0}\rangle$ of two neighboring triplet states, which alter the sign of $(-1)^{M_{\text {odd }}}$. Note that they coincide with similar matrix elements of the Haldane chain, in (nonpositive) basis formed by the states $(-1)^{M_{\text {odd }}}\left|m_{1}\right\rangle \otimes \ldots \otimes\left|m_{N}\right\rangle \underline{14}^{14}$ So, the composite spin part of the Hamiltonian is nonpositive in the basis (4).

The matrix elements produced by the antisymmetric local terms of Hamiltonian (17) have been considered already in Ref. 21. In terms of lowering-raising operators $S^{(a) \pm}=S^{(a) x} \pm i S^{(a) y}$, each such term reads $\left(S_{l}^{(a)+} S_{l+1}^{(a)-}+S_{l}^{(a)-} S_{l+1}^{(a)+}\right) / 2+S_{l}^{(a) z} S_{l+1}^{(a) z}$. In contrary to the symmetric case, the antisymmetrized spin operators mix triplet and singlet states. Their nonzero matrix elements are: ${ }^{26}$

$$
\begin{aligned}
& \left\langle 0\left|S^{(a)+}\right|-1\right\rangle=\sqrt{2}, \quad\left\langle 1\left|S^{(a)+}\right| 0\right\rangle=-\sqrt{2} \\
& \left\langle\tilde{0}\left|S^{(a) z}\right| 0\right\rangle=1 .
\end{aligned}
$$


Using the above equations and the definition of basic states (4), it is easy to check that

$$
\begin{gathered}
\left\langle\ldots, 0_{l}, 0_{l+1}, \ldots\left|S_{l}^{(a) \mp} S_{l+1}^{(a) \pm}\right| \ldots, \pm 1_{l}, \mp 1_{l+1}, \ldots\right\rangle \\
=-2(-1)^{\left[N_{0} / 2\right]+N_{0 \tilde{0}}+M_{\text {odd }}-\left[N_{0}^{\prime} / 2\right]-N_{0 \tilde{0}}^{\prime}-M_{\text {odd }}^{\prime}}=-2,
\end{gathered}
$$

where the unchanged sites are replaced by dots. The quantum numbers of bra- and -ket states are mentioned, respectively, without and with primes. Indeed, according to the definition of $N_{0 \tilde{0}}$, the difference $N_{0 \tilde{0}}-N_{0 \tilde{0}}^{\prime}$ in (10) is an even number. Also, $N_{0}^{\prime}=N_{0}+2$ and $M_{\text {odd }}=M_{\text {odd }}^{\prime} \pm 1$ depending on whether $l$ is even or odd. Therefore, the exponent in (10) is an even number, and the equation is true. The next nontrivial matrix element is also negative. Namely,

$$
\begin{array}{r}
\left\langle\ldots, 0_{l}, \pm 1_{l+1}, \ldots\left|S_{l}^{(a) \mp} S_{l+1}^{(a) \pm}\right| \ldots, \pm 1_{l}, 0_{l+1}, \ldots\right\rangle \\
=2(-1)^{M_{\text {odd }}-M_{\text {odd }}^{\prime}}=2(-1)^{ \pm 1}=-2
\end{array}
$$

because the quantum numbers $N_{00}$ and $N_{0}$ are the same for both states.

In contrast, the $z$ projections of antisymmetrized spin operators preserve the quantum number $M_{\text {odd }}$. They produce negative matrix elements too:

$$
\begin{aligned}
& \left\langle\ldots, 0_{l}, 0_{l+1}, \ldots\left|S_{l}^{(a) z} S_{l+1}^{(a) z}\right| \ldots, \tilde{0}_{l}, \tilde{0}_{l+1}, \ldots\right\rangle \\
= & (-1)^{N_{0 \tilde{0}}-N_{0 \tilde{0}}^{\prime}+\left(N_{0}-N_{0}^{\prime}\right) / 2}=(-1)^{\text {even-1}}=-1
\end{aligned}
$$

and

$$
\begin{array}{r}
\left\langle\ldots, \tilde{0}_{l}, 0_{l+1}, \ldots\left|S_{l}^{(a) z} S_{l+1}^{(a) z}\right| \ldots, 0_{l}, \tilde{0}_{l+1}, \ldots\right\rangle \\
=(-1)^{N_{0 \tilde{0}}-N_{0 \tilde{o}}^{\prime}}=(-1)^{1}=-1 .
\end{array}
$$

The expressions (10), (11), (12), (13) together with conjugate ones constitute the full set of nontrivial matrix elements of Hamiltonian (77) generated by $J^{a}$ terms.

Finally, consider the off-diagonal terms, which are responsible for four-spin cyclic exchange. The operator $P_{l, l+1}$ just permutes two adjacent rung states. At the same time, $P_{l, l+1}^{\times}$is a signed permutation: While permuting the singlet with a triplet state it produces an additional minus sign. Therefore, the difference $P_{l, l+1}^{=}-P_{l, l+1}^{\times}$ vanishes if the spins of both rungs are the same. If their spins differ, this operator just permutes them multiplying by 2 . Then, using the definition (4) of basic states, we obtain

$$
\begin{array}{r}
\left\langle\ldots, t_{l}, 0_{l+1}, \ldots\left|P_{l, l+1}^{\overline{\bar{l}}}-P_{l, l+1}^{\times}\right| \ldots, 0_{l}, t_{l+1}, \ldots\right\rangle \\
=2(-1)^{M_{\text {odd }}-M_{\text {odd }}^{\prime}+N_{0 \tilde{o}}-N_{0 \tilde{o}}^{\prime}}=-2,
\end{array}
$$

where $t=\tilde{0}, \pm 1$ is any triplet state. Indeed, in the sign factor above, $M_{\text {odd }}=M_{\text {odd }}^{\prime}$ and $N_{0 \tilde{0}}-N_{0 \tilde{0}}^{\prime}=1$ for $t=\tilde{0}$. For $t= \pm 1, N_{0 \tilde{0}}=N_{0 \tilde{0}}^{\prime}$ and $\left|M_{\text {odd }}-M_{\text {odd }}^{\prime}\right|=1$. Together with the conjugate element, this is a sole nonvanishing off-diagonal matrix element produced by the four-spin cyclic exchange term.

According to the constraints (2) imposed on the couplings, the coefficients $J_{l}^{s}, J_{l}^{a}, K_{l}$ in (7) are positive. This finishes the proof that the ladder Hamiltonian has no positive off-diagonal element in the basis (4).

Due to the spin and reflection symmetries, the Hamiltonian is invariant on each $(M, \sigma)$ subspace, all states of which have $S^{z}=M$ and $\sigma= \pm 1$ quantum numbers. Any two basic states within the same subspace are connected at least by two-spin interaction terms of the Hamiltonian, as can be easily verified by induction. $\stackrel{21}{=}$ So, we can apply the Perron-Frobenius theorem 27 to the matrix of the Hamiltonian restricted to any $(M, \sigma)$ subspace. As a result, the lowest energy state there (usually called a relative ground state) is unique and is a positive superposition of all basic states:

$$
|\Omega\rangle_{M, \sigma}=\sum_{\left|m_{1}, \ldots, m_{N}\right\rangle \in(M, \sigma)} \omega_{m_{1} \ldots m_{N}}\left|m_{1}, \ldots, m_{N}\right\rangle,
$$

where $\omega_{m_{1} \ldots m_{N}}>0$. The uniqueness implies that this state must have a certain value of spin $S_{M, \sigma}$, which can be obtained by comparing with the similar state of the Haldain chain. The last model corresponds to the restriction of the composite spin model $\sum_{l} \mathbf{S}_{l}^{(s)} \cdot \mathbf{S}_{l+1}^{(s)}$ on the states with triplets on all rungs. In fact, all such type states from (4) form a nonpositive basis for the Hal-


subspace is a positive superposition of all basic states and has the highest possible spin value, i.e., $|M|$, except $M=0$ and odd $N$ case when its spin is one $\underline{\underline{14}}$ Therefore, for $\sigma=1$, both states $|\Omega\rangle_{M, \sigma}$ and $|\Omega\rangle_{M}$ overlap, and, hence, must have the same spin. Similarly, the restriction of composite spin model to the subspace of states with one singlet frozen at the last rung corresponds to the Haldane chain with $N-1$ sites. So, for $\sigma=-1$, the spin of (15) coincides with the spin of the corresponding state of the Haldane chain having one site less. Therefore, the spin of the relative ground state (15) is $|M|$ except $M=0$ and $\sigma=(-1)^{N-1}$ case when it equals one.

Now we are ready to finish the proof of our main result. For $\sigma=(-1)^{N}$, the relative ground states $|\Omega\rangle_{ \pm S, \sigma}$ are, correspondingly, the highest and lowest states of a unique spin- $S$ multiplet, which has the minimum energy $E_{\sigma}(S)$ among all spin- $S$ states with parity $\sigma$. The $(S, \sigma)$ subspace contains a representative from any multiplet with $S^{\prime} \geq S$ and parity $\sigma$. Together with the uniqueness condition, this implies that $E_{\sigma}\left(S^{\prime}\right)$ must be higher than $E_{\sigma}(S)$ for all $S^{\prime}>S$. Consequently, $E_{\sigma}(S)$ is a monotone increasing function of $S$, and the ground state in $\sigma=(-1)^{N}$ sector is a nondegenerate spin singlet. For $\sigma=(-1)^{N-1}$, the states $|\Omega\rangle_{ \pm S, \sigma}$ are the highest and lowest states only if $S \geq 1$. Therefore, $E_{\sigma}(S)$ monotonically increases in this region. Note that the states $|\Omega\rangle_{ \pm 1, \sigma}$ and $|\Omega\rangle_{0, \sigma}$, which have the lowest energy in $\sigma=(-1)^{N-1}$ sector, form a spin triplet.

Consider separately the limiting case of $J_{l}^{\|}=2 K_{l}$ when the $J^{a}$ terms in (7) are absent. Then $\sum_{l}\left(\mathbf{S}_{l}^{(s)}\right)^{2}$ commutes with the Hamiltonian and with the total spin operator $\stackrel{12}{12}$ As a result, the symmetry group $\mathrm{SU}(2) \times Z_{2}$ expands to $\mathrm{SU}(2) \times \mathrm{U}(1)=\mathrm{U}(2)$. The $\mathrm{U}(1)$ symmetry reflects the invariance under the duality transformation 
and results in the conservation of singlet number $N_{0}$ Any $(M, \sigma)$ subspace splits into invariant subspaces with fixed singlet number obeying $(-1)^{N_{0}}=\sigma$. It is easy to see that the Hamiltonian is connected on every such subspace. Therefore, the relative ground state there is unique and is a positive superposition of all basic states (4) with $S^{z}=M$ and $N_{0}$ rung singlets. Again, comparing it with the action of the composite spin model on the states with singlets on the last $N_{0}$ rungs, one can conclude that the relative ground state has the highest possible spin value, except $M=0$ and odd $N-N_{0}$ case when it is a triplet state. The antiferromagnetic ordering of energy levels takes place independently in any sector with fixed singlet number and corresponds to the similar rule for the Haldane chain with $N-N_{0}$ spins.

For appropriate values of couplings, the results of this paper remain true, if diagonal interactions $\mathbf{S}_{1, l} \cdot \mathbf{S}_{2, l+1}+$ $\mathbf{S}_{1, l+1} \cdot \mathbf{S}_{2, l}$ are included in the Hamiltonian (1). Similarly, one can consider a more general biquadratic interaction of type $K_{l}^{\prime}\left(\mathbf{S}_{1, l} \cdot \mathbf{S}_{1, l+1}\right)\left(\mathbf{S}_{2, l} \cdot \mathbf{S}_{2, l+1}\right)+4 K_{l}\left(\mathbf{S}_{1, l}\right.$. $\left.\mathbf{S}_{2, l}\right)\left(\mathbf{S}_{1, l+1} \cdot \mathbf{S}_{2, l+1}\right)-4 K_{l}\left(\mathbf{S}_{1, l} \cdot \mathbf{S}_{2, l+1}\right)\left(\mathbf{S}_{2, l} \cdot \mathbf{S}_{1, l+1}\right)$ with arbitrary couplings $K_{l}^{\prime}$ because the first term in the sum is diagonal in the basis (3), (4).

\section{Acknowledgments}

The author is grateful to V. Ohanyan for useful discussions. This work was supported by grants UC-06/07, INTAS-05-7928, ANSEF-1386-PS and the Artsakh Ministry of Science \& Education.
* Electronic address: hakob@yerphi.am

1 M. Roger, J. Phys. Chem. Sol. 66, 1412 (2005), cond-mat/0504141.

2 G. Misguich and C. Lhuillier, in Frustrated Spin Systems, edited by H. T. Diep (World Scientific, Singapore, 2005), pp. 229-307, cond-mat/0310405

3 M. Takahashi, J. Phys. C 10, 1289 (1977); A. H. MacDonald, S. M. Girvin, and D. Yoshioka, Phys. Rev. B 41, 2565 (1990); ibid. 37, 9753 (1988); A. L. Chernyshev, D. Galanakis, P. Phillips, A. V. Rozhkov, and A.M. S. Tremblay, Phys. Rev. B 70, 235111 (2004).

4 D. J. Thouless, Proc. Phys. Soc. London 86, 893 (1965); M. Roger, J. H. Hetherington, and J. M. Delrieu, Rev. Mod. Phys. 55, 1 (1983), and references therein.

5 M. Roger and J. M. Delrieu, Phys. Rev. B 39, 2299 (1989).

${ }^{6}$ E. Dagotto, Rep. Prog. Phys. 62, 1525 (1999); E. Dagotto and T. M. Rice, Science 271, 618 (1996).

7 S. Brehmer, H.-J. Mikeska, M. Muller, N. Nagaosa, and S. Uchida, Phys. Rev. B 60, 329 (1999); T. S. Nunner, P. Brune, T. Kopp, M. Windt, and M. Grüninger, Phys. Rev. B 66, 180404(R) (2002); N. Haga, S. I. Suga, Phys. Rev. B 67, 134432 (2003); K. P. Schmidt, A. Gössling, U. Kuhlmann, C. Thomsen, A. Löffert, C. Gross, and W. Assmus, Phys. Rev. B 72, 094419 (2005); A. Gössling, U. Kuhlmann, C. Thomsen, A. Löffert, C. Gross, and W. Assmus, Phys. Rev. B 67, 052403 (2003); M. Matsuda, K. Katsumata, R. S. Eccleston, S. Brehmer, and H.J. Mikeska, Phys. Rev. B 62, 8903 (2000); J. Appl. Phys. 87, 6271 (2000).

${ }^{8}$ K. P. Schmidt and G. S. Uhrig, Mod. Phys. Lett. B 19, 1179 (2005).

9 D. Allen, F. H. L. Essler, and A. A. Nersesyan, Phys. Rev. B 61, 8871 (2000); X. Wang, Mod. Phys. Lett. B 14, 327 (2000); E. H. Kim, G. Fáth, J. Sólyom, and D. J. Scalapino, Phys. Rev. B 62, 14965 (2000); T. Hakobyan, J. H. Hetherington, and M. Roger, Phys. Rev. B 63, 144433 (2001); B. W. Ramakko and M. Azzouz, Phys. Rev. B 76, 064419 (2007); Can. J. Phys. 86, 509 (2008); E. H. Kim, Ö. Legeza, and J. Sólyom, Phys. Rev. B 77, 205121 (2008).

10 A. A. Nersesyan and A. M. Tsvelik, Phys. Rev. Lett. 78, 3939 (1997); M. Müller, T. Vekua, and H.-J. Mikeska,
Phys. Rev. B 66, 134423 (2002); A. Läuchli, G. Schmid, and M. Troyer, Phys. Rev. B 67, 100409(R) (2003); Y. Honda and T. Horiguchi, cond-mat/0106426 (unpublished); K. Hijii and K. Nomura Phys. Rev. B 65, 104413 (2002); M. Sato, Phys. Rev. B 76, 054427 (2007); T. Hikihara and Sh. Yamamoto, J. Phys. Soc. Jpn. 77, 014709 (2008).

11 T. Hikihara, T. Momoi, and Xiao Hu, Phys. Rev. Lett. 90, 087204 (2003).

12 T. Momoi, T. Hikihara, M. Nakamura, and Xiao Hu, Phys. Rev. B 67, 174410 (2003).

13 P. Lecheminant and K. Totsuka, Phys. Rev. B 74, 224426 (2006).

14 E. H. Lieb and D. Mattis, J. Math. Phys. 3, 749 (1962); E. H. Lieb, Phys. Rev. Lett. 62, 1201 (1989).

15 E. H. Lieb and D. Mattis, Phy. Rev. 125, 164 (1962).

16 T. Hakobyan, Nucl. Phys. B 699, 575 (2004).

17 B. Nachtergaele, W. Spitzer, and Sh. Starr, J. Stat. Phys. 116, 719 (2004); B. Nachtergaele and Sh. Starr, Phys. Rev. Lett. 94, 057206 (2005)

18 J. Richter, K. Retzlaff, A. Voigt, and N. B. Ivanov, J. Magn. Magn. Mater. 140-144, 1611 (1995); J. Richter, N. B. Ivanov, A. Voigt, and K. Retzlaff, J. Low Temp. Phys. 99, 363 (1995).

19 Y. J. Liu, Y. C. Chen, M. F. Yang, and C. D. Gong, Phys. Rev. B 66, 024403 (2002).

20 J. Schnack, H.-J. Schmidt, J. Richter, and J. Schulenburg, Eur. Phys. J. B 24, 475 (2001); J. Schnack, Lect. Notes Phys. 645, 155-194 (2004).

21 T. Hakobyan, Phys. Rev. B 75, 214421 (2007).

22 F. D. M. Haldane, Phys. Rev. Lett. 50, 1153 (1983).

23 V. Gritsev, B. Normand, and D. Baeriswyl, Phys. Rev. B 69, 094431 (2004).

${ }^{24}$ Y. Xian, Phys. Rev. B 52, 12485 (1995).

25 J. Solyom and J. Timonen, Phys. Rev. B 34, 487 (1986); 39, 7003 (1989); 40, 7150 (1989).

26 J.-B. Fouet, F. Mila, D. Clarke, H. Youk, O. Tchernyshyov, P. Fendley, and R. M. Noack, Phys. Rev. B 73, 214405 (2006).

27 P. Lancaster, Theory of Matrices (Academic, New York, 1969). 